\title{
A General Transfer-Function Approach to Noise Filtering in Open-Loop Quantum Control
}

\author{
Gerardo A. Paz-Silva and Lorenza Viola \\ Department of Physics and Astronomy, Dartmouth College, 6127 Wilder Laboratory, Hanover, New Hampshire, USA
}

(Dated: October 5, 2018)

\begin{abstract}
We present a general transfer-function approach to noise filtering in open-loop Hamiltonian engineering protocols for open quantum systems. We show how to identify a computationally tractable set of fundamental filter functions, out of which arbitrary transfer filter functions may be assembled up to arbitrary high order in principle. Besides avoiding the infinite recursive hierarchy of filter functions that arises in general control scenarios, this fundamental filter-functions set suffices to characterize the error suppression capabilities of the control protocol in both the time and frequency domain. We prove that the resulting notion of filtering order reveals conceptually distinct, albeit complementary, features of the controlled dynamics as compared to the order of error cancellation, traditionally defined in the Magnus sense. Examples and implications are discussed.
\end{abstract}

PACS numbers: 03.67.Pp, 03.65.Yz, 03.67.Lx, 07.05.Dz

Hamiltonian engineering via open-loop quantum control provides a versatile and experimentally validated framework for manipulating the dynamics of a broad class of open quantum systems [1]. Applications range from dynamical decoupling (DD), composite pulse sequences and dynamically corrected quantum gates (DCGs), to noise spectroscopy and quantum simulation - see e.g. [2,8] for recent contributions. In this context, generalized transfer filter function (FF) techniques motivated by control engineering are providing an increasingly important tool for understanding the dynamical response of the target system in Fourier space and for quantitatively analyzing the control performance [9-12]. In particular, this formalism has proved remarkably successful in predicting operational fidelities for a variety of control settings in recent trapped-ion experiments [13], as long as noise is sufficiently weak for low-order approximations to be viable.

From a control-theory standpoint, a filtering approach to dynamical error suppression is desirable for a variety of reasons. Besides providing an open-loop counterpart to the transfer-function perspective that is central to both classical and quantum feedback networks [14], FF techniques allow, when available, for a substantially more efficient analysis of the underlying noisy dynamics than direct simulation [15, 16]. Furthermore, unlike traditional time-dependent perturbative approaches (such as the Magnus expansion [1, 17]), a frequency-space picture may open up new possibilities for tailoring control synthesis and optimization to specific spectral features of the noise. The existing FF framework suffers, however, from severe limitations. Even if formal expressions for gate fidelity may be given based on an infinite recursive hierarchy of generalized FFs [12], higher-order FFs become rapidly intractable. Thus, explicit calculations have largely focused thus far on single-qubit controlled dynamics in the presence of classical noise, by truncating this recursion to the lowest order and additionally exploiting Gaussian noise statistics. As experiments are revealing the importance of higher-order terms outside the weak-noise regime [13], and are rapidly progressing toward coupled-qubit systems and/or more complex, non-Gaussian error models [18], overcoming these limitations becomes imperative for further progress. An additional short- coming arises from the fact that only in very special situations where a single FF suffices to characterize the controlled dynamics, one may unambiguously identify the "order of error suppression" of the protocol, associated to the order of cancellation in the Magnus expansion, with its "filtering order", determined by the low-frequency behavior of the FF [4, 12]. Thus, the present theory does not lend itself to the identification of relevant filtering notions for general control scenarios.

In this Letter, we introduce a filtering framework for openloop dynamical control based on an easily computable set of fundamental filter functions (FFFs). FFFs replace the unwieldy recursive construction of existing schemes by allowing for arbitrary high-order FFs to be expressed as simple linear combinations of products of elements in the set. We show that FFFs encode all the relevant information about the error-suppressing capabilities of a control protocol in both the time and frequency domain. In addition, they suffice to rigorously characterize the (minimum) order of cancellation vs. filtering that can be guaranteed when no specific assumptions are made on the noise model - other than the bath operators be norm-bounded, in the same spirit of DCG theory [6] and non-Markovian quantum error correction [19]. We prove that cancellation and filtering are inequivalent notions, with highorder cancellation in the Magnus sense not implying highorder filtering in general, and with both notions being a priori equally significant for assessing the control performance. Our results provide a firm foundation for recent analyses where this inequivalence has manifested in the context of compositepulse and Walsh-modulated protocols [13, 15], as well as a new perspective on dynamical error control strategies, with potential implications for quantum fault tolerance.

Control-theoretic setting.- We consider a general finitedimensional open quantum system $S$ coupled to an uncontrollable environment (bath) $B$, whose free evolution is described by a joint Hamiltonian of the form $H(t)=H_{S}+H_{S B}(t)$, with respect to the interaction picture associated to the physical bath Hamiltonian $H_{B}$. Open-loop control is introduced via a time-dependent Hamiltonian $H_{\text {ctrl }}(t)$ acting on $S$ alone, with the controlled dynamics being represented in terms of an intended plus error component, namely, $H(t)+H_{\mathrm{ctrl}}(t) \equiv$ 
$H_{0}(t)+H_{e}(t)$, and evolution under $H_{0}(t)$ over time $T$ yielding the desired unitary gate (say, $Q$ ) on $S$. In addition to the system-bath coupling $H_{S B}(t)$ and non-Markovian control errors $H_{\mathrm{ctrl}, \mathrm{e}}(t)$, the error Hamiltonian $H_{e}(t)$ may or may not include $H_{S}$, depending on whether the objective is noisesuppressed storage (DD, in which case $Q=\mathbb{I}_{S}$ ) or a non-trivial logic gate (such as in DCGs or composite pulses) [6].

The effect of $H_{e}(t)$ may be isolated by expressing the total (interaction-picture) propagator for evolution over time $T$ as $U(T)=U_{0}(T) \tilde{U}_{e}(T) \equiv Q \tilde{U}_{e}(T)$, where the "error propagator" $\tilde{U}_{e}(t)=\mathcal{T} \exp \left[-i \int_{0}^{t} \tilde{H}_{e}(s) d s\right]$ describes evolution in the toggling-frame defined by the ideal Hamiltonian $H_{0}(t)$ (e.g., in a drift-less, ideal-control setting, $H_{S}=0=H_{\text {ctrl, e }}(t)$, hence $\left.H_{0}(t)=H_{\text {ctrl }}(t), \tilde{H}_{e}(t)=U_{\text {ctrl }}(t)^{\dagger} H_{e}(t) U_{\text {ctrl }}(t)\right)$. Without loss of generality, we may write $H_{e}(t)=\sum_{u} O_{u} \otimes B_{u}(t)$, with respect to Hermitian traceless operators $\left\{O_{u}\right\}$ which, together with $\mathbb{I}_{S}$, form an operator basis on $S$. The limit of a classical noise source may be recovered by letting $B(t)=\beta(t) \mathbb{I}_{B}$, with $\beta(t)$ being a classical stochastic process. Accordingly,

$$
\tilde{H}_{e}(t)=U_{0}(t)^{\dagger} H_{e}(t) U_{0}(t) \equiv \sum_{u, v} y_{u v}(t) O_{v} \otimes B_{u}(t),
$$

where the (target-dependent) "control matrix" $y_{u v}(t)$ encapsulates the intended effect of the control. Dynamical error suppression aims to perturbatively minimize the sensitivity of the controlled evolution to $H_{e}(t)$, by making $\tilde{U}_{e}(T)$ as close as possible to a "pure-bath" evolution. Specifically,

$$
\tilde{U}_{e}(T)=e^{\sum_{\alpha=1}^{\infty} \Omega_{\alpha}(T)} \equiv e^{-i T\left(H_{S B}^{\mathrm{eff}}(T)+H_{B}^{\mathrm{eff}}(T)\right)}
$$

can be obtained via a Magnus series expansion [17], where the $\alpha$-th order Magnus term $\Omega_{\alpha}(T)$ is a time-ordered integral of a function of $\alpha$-th order nested commutators of $\tilde{H}_{e}\left(t_{j}\right)$, $j \in\{1, \ldots, \alpha\}$, over the volume $V_{\alpha} \equiv\left\{0 \leq t_{\alpha} \leq t_{\alpha-1} \leq \ldots \leq\right.$ $\left.t_{2} \leq t_{1} \leq T\right\}$, and the second equality in Eq. (2) defines the relevant effective Hamiltonian and (Hermitian) "error action" operator $\Omega_{e}(T) \equiv H^{\text {eff }}(T) T=i \sum_{\alpha} \Omega_{\alpha}(T)$ [6].

A control protocol $y_{u v}(t)$ is said to achieve cancellation $\operatorname{order}(\mathrm{CO}) \delta \geq 1$ if the norm of $\Omega_{e}(T)$ (up to pure-bath terms) is reduced, so that the leading-order correction mixing $S$ and $B$ scales as $\left\|T H_{S B}^{\text {eff }}(T)\right\|=\mathcal{O}\left(T^{\delta+1}\right)$. The $C O$ coincides with the decoupling order in the paradigmatic DD case [2,-4, 20].
Generalized filter functions. - In their most basic form, generalized FFs may be defined by seeking a representation of the control action in frequency space directly at the effective Hamiltonian level of Eq. (2). By expressing each of the $\tilde{H}_{e}\left(t_{j}\right)$ in the $\alpha$-th Magnus term $\Omega_{\alpha}(T)$ using Eq. 11 , followed by appropriate index relabeling, the latter reads

$$
\begin{aligned}
\Omega_{\alpha}(T)= & \sum_{\vec{u}, \vec{v}} \int_{V_{\alpha}} d^{\alpha} \vec{t} \sum_{p \in \Pi\left[\left\{t_{j}\right\}\right]} f^{(p)}\left(\left\{y_{[\alpha]}\right\}\right) O_{v_{1}} \cdots O_{v_{\alpha}} \\
& \otimes B_{u_{1}}\left(p\left(t_{1}\right)\right) \cdots B_{u_{\alpha}}\left(p\left(t_{\alpha}\right)\right),
\end{aligned}
$$

where $\vec{u} \equiv\left(u_{1}, \ldots, u_{\alpha}\right), \Pi\left[\left\{t_{j}\right\}\right]$ denotes the set of permutations of the labels $\left\{t_{j}\right\}$, and the function $f^{(p)}$ depends on products of $\alpha$ control matrix elements, $y_{[\alpha]} \equiv$ $y_{u_{1} v_{1}}\left(p\left(t_{1}\right)\right) \cdots y_{u_{\alpha} v_{\alpha}}\left(p\left(t_{\alpha}\right)\right)$. Upon writing the bath variables in terms of their frequency-Fourier transform, $B_{u}(t) \equiv$ $\int_{-\infty}^{\infty}(d \omega / 2 \pi) e^{i \omega t} B_{u}(\omega)$, and assuming sufficient regularity for the relevant integrals to converge, the $\alpha$-th order Magnus term takes then the desired form:

$$
\begin{aligned}
\Omega_{\alpha}(T) & =-i \sum_{\vec{u}, \vec{v}} \int \frac{d^{\alpha} \vec{\omega}}{(2 \pi)^{\alpha}} G_{\vec{u} \vec{v}}^{(\alpha)}(\vec{\omega}, T) \\
& \times O_{v_{1}} \cdots O_{v_{\alpha}} \otimes B_{u_{1}}\left(\omega_{1}\right) \cdots B_{u_{\alpha}}\left(\omega_{\alpha}\right),
\end{aligned}
$$

where the $\alpha$-th order generalized $F F G_{\vec{u}, \vec{v}}^{(\alpha)}(\vec{\omega}, T)$ describes the extent to which the applied control "filters" the effect of $O_{v_{1}} \cdots O_{v_{\alpha}} \otimes B_{u_{1}}\left(\omega_{1}\right) \cdots B_{u_{\alpha}}\left(\omega_{\alpha}\right)$ in $\Omega_{\alpha}(T)$.

In practice, one may have access to quantities that depend on the reduced (or ensemble-averaged, for classical noise) dynamics of the system alone. The time-evolved reduced state, $\rho_{S}(T) \equiv \sum_{\ell, \ell^{\prime}} \rho_{\ell \ell^{\prime}}(T)|\ell\rangle\left\langle\ell^{\prime}\right|$ with respect to an orthonormal basis on $S$, may be computed by averaging over the bath variables. Choosing a basis where the target gate $Q$ is diagonal, with $Q|\ell\rangle=q_{\ell}|\ell\rangle$, and assuming as usual that the initial state has the factorized form $\rho_{S B}(0) \equiv \rho_{S}(0) \otimes \rho_{B}$, we thus need

$$
\begin{aligned}
& \rho_{\ell \ell^{\prime}}(T)=q_{\ell}^{*} q_{\ell^{\prime}}\left\langle\ell\left|\operatorname{Tr}_{B}\left[\tilde{U}_{e}(T) \rho_{S}(0) \otimes \rho_{B} \tilde{U}_{e}(T)^{\dagger}\right]\right| \ell^{\prime}\right\rangle \\
& =q_{\ell}^{*} q_{\ell^{\prime}} \sum_{m, m^{\prime}} \rho_{m, m^{\prime}}(0) \operatorname{Tr}_{B}\left[\left\langle\ell\left|\tilde{U}_{e}(T)\right| m\right\rangle \rho_{B}\left\langle m^{\prime}\left|\tilde{U}_{e}(T)^{\dagger}\right| \ell^{\prime}\right\rangle\right] .
\end{aligned}
$$

Formally, we may Taylor-expand the error propagator given in Eq. 22, say, $\tilde{U}_{e}(T)=\sum_{r=0}^{\infty}\left(-i \Omega_{e}(T)\right)^{r} / r$ !. Then the trace over $B$ in the above expression is given by an infinite sum of terms each of which, fixing $r$ and $r^{\prime}$ (in the corresponding expansion of $\left.\tilde{U}_{e}(T)^{\dagger}\right)$, and taking advantage of Eq. (3), may be recognized to have the following symbolic structure:

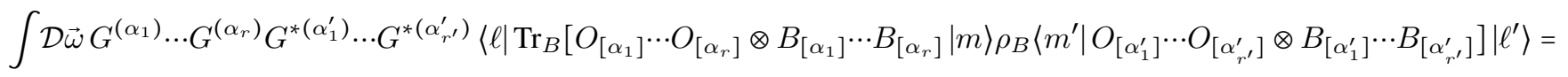

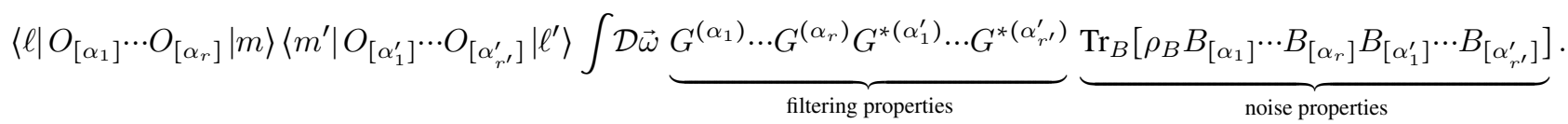

Here, the additional indexes and arguments of each generalized FF $G^{\left(\alpha_{j}\right)} \equiv G_{\vec{u}_{\alpha_{j}} \vec{v}_{\alpha_{j}}}^{\left(\alpha_{j}\right)}\left(\omega_{1}, \ldots \omega_{\alpha_{j}}, T\right)$ have been left im- 
plicit, we have used shorthand notations like $O_{\left[\alpha_{j}\right]}\left(B_{\left[\alpha_{j}^{\prime}\right]}\right)$ to denote strings of system (bath) operators of length $\alpha_{j}\left(\alpha_{j}^{\prime}\right)$, and $\int \mathcal{D} \vec{\omega}$ denotes a multidimensional integral over all the relevant frequency variables. The main point is that in each term it is possible to clearly identify a filtering action on quantities that depend solely on "high-order moments" of the noise in frequency space, taken (in the quantum case) with respect to the initial state of $B$. In fact, such bath expectations may be related to the (high-order) noise power spectra given by the Fourier transform of the appropriate cumulant. Following [21, 22], we may define the $k$-th order noise power spectrum:

$$
\begin{aligned}
S_{\vec{u}}^{(k)}(\vec{\omega}) & =\int_{-\infty}^{\infty} d^{k} \vec{t} e^{-i \vec{\omega} \cdot \vec{t}} \mathcal{C}\left(B_{u_{1}}\left(t_{1}\right) \cdots B_{u_{k}}\left(t_{k}\right)\right) \\
& =\mathcal{C}\left(B_{u_{1}}\left(\omega_{1}\right) \cdots B_{u_{k}}\left(\omega_{k}\right)\right),
\end{aligned}
$$

where $\mathcal{C}\left(B_{u_{1}}\left(t_{1}\right) \cdots B_{u_{k}}\left(t_{k}\right)\right)$ is the $k$-th order (generalized) cumulant with respect to $\rho_{B}$, that is, computed from the appropriate moment expansion by taking expectations with respect to $\rho_{B}$ and retaining operator ordering [23, 24].

In this way, one may make contact with the standard FF formalism [4, 10-12]. The simplest setting is provided by "bang-bang" DD on a single qubit exposed to either classical zero-mean, stationary Gaussian phase noise or spin-boson dephasing from a thermal bath. Without loss of generality, we can let $\tilde{H}_{e}(t)=y(t) \sigma_{z} \otimes B(t)$, with the "switching" function $y(t)= \pm 1$ being determined by the applied pulse sequence $p$. The Gaussian statistics allows for the infinite expansion determining the coherence matrix element $\rho_{01}(T) \equiv$ $\rho_{01}(0) e^{-\chi_{p}(T)}$ to be exactly re-summed [22], with $\chi_{p}(T)=$ $2 \int_{-\infty}^{\infty} d \omega /(2 \pi) G^{(1)}(\omega, T) G^{(1)}(-\omega, T) S^{(2)}(\omega,-\omega)$, where $G^{(1)}(\omega, T)=\int_{0}^{T} d t y(t) e^{i \omega t}$ and, thanks to stationarity, $S^{(2)}\left(\omega, \omega^{\prime}\right)=2 \pi \delta\left(\omega+\omega^{\prime}\right) S(\omega)$. Thus, the "traditional" FF is recovered as $F_{p}(\omega, T) \equiv \omega^{2} G^{(1)}(\omega, T) G^{(1)}(-\omega, T)$. By construction, if the FF is small around some frequency $\omega_{0}$, the contribution of the noise at that frequency is suppressed. This may be formalized by considering the Taylor expansion of the $\mathrm{FF}$ around $\omega_{0}$. For DD, one is interested in $\omega_{0}=0$, yielding $F_{p}(\omega, T) \propto(\omega T)^{2(\delta+1)}$ [4, 11]. Thus, if a filtering order (FO) $\phi$ is defined by the frequency scaling of $G^{(1)}$ as $|\omega| \rightarrow 0$, $\mathrm{CO}$ and FO coincide in this exactly solvable model.

Fundamental filter functions and filtering order-- Away from special scenarios where a single FF fully characterizes the cancellation and filtering properties of the applied control, evaluating arbitrary generalized FFs $G_{\vec{u}, \vec{v}}^{(\alpha)}(\vec{\omega}, T)$ may seem beyond reach in general. Our key insight is to realize that, despite their complexity, generalized FFs share a common structure, determined by a still infinite but easily computable FF set. Let FFFs of order $\alpha$ be defined as follows:

$$
F_{\vec{u} \vec{v}}^{(\alpha)}(\vec{\omega}, T) \equiv(-i)^{\alpha} \int_{V_{\alpha}} d^{\alpha} \vec{t} \prod_{j=1}^{\alpha}\left(y_{u_{j} v_{j}}\left(t_{j}\right) e^{i \omega_{j} t_{j}}\right),
$$

where $V_{\alpha}$ is the integration volume previously defined. Let us also introduce the notation $s_{k} \equiv \sum_{r=1}^{k} \alpha_{r}$ and $\vec{u}_{\left[s_{k-1}, s_{k}\right]} \equiv$ $\left(u_{s_{k-1}+1}, \cdots, u_{s_{k}}\right)$. Our main result is then the following:

Theorem 1. Arbitrary generalized FFs of order $\alpha, \alpha=$
$1, \ldots, \infty$, may be exactly represented in terms of FFFs of order at most $\alpha$ :

$$
\begin{aligned}
& -i G_{\vec{u} \vec{v}}^{(\alpha)}(\vec{\omega}, T)=F_{\vec{u} \vec{v}}^{(\alpha)}(\vec{\omega}, T) \\
& -\sum_{j=2}^{\alpha} \frac{(-1)^{j}}{j} \sum_{\substack{\sum_{r=1}^{j} \alpha_{r}=\alpha \\
\left\{\alpha_{r}>0\right\}}} \prod_{k=1}^{j} F_{\vec{u}_{\left[s_{k-1}, s_{k}\right]} \vec{v}_{\left[s_{k-1}, s_{k}\right]}^{\left(\alpha_{k}\right)}\left(\vec{\omega}_{\left[s_{k-1}, s_{k}\right]}, T\right) .}
\end{aligned}
$$

Proof. By using the Dyson series expansion, the error propagator $\tilde{U}_{e}(T)$ in Eq. (2) is given by $\tilde{U}_{e}(T)=$ $\mathbb{I}+\sum_{\alpha=1}^{\infty} \mathcal{D}_{\alpha}(T)$, where the $\alpha$-th Dyson term is a timeordered integral over $V_{\alpha}$ of a function of $\alpha$ products of $\tilde{H}_{e}\left(t_{j}\right)$. In frequency space, the latter $\operatorname{reads} \mathcal{D}_{\alpha}(T)=$ $\sum_{\vec{u}, \vec{v}} \int \frac{d^{\alpha} \vec{\omega}}{(2 \pi)^{\alpha}} F_{\vec{u} \vec{v}}^{(\alpha)}(\vec{\omega}, T) O_{v_{1}} \cdots O_{v_{\alpha}} \otimes B_{u_{1}}\left(\omega_{1}\right) \cdots B_{u_{\alpha}}\left(\omega_{\alpha}\right)$. One then makes use of the well-known expression relating Dyson and Magnus terms of a given order [17], namely, $\Omega_{1}=$ $\mathcal{D}_{1}, \Omega_{\alpha}=\mathcal{D}_{\alpha}-\sum_{j=2}^{\alpha}\left((-1)^{j} / j\right) \sum_{\alpha_{1}+\ldots \alpha_{j}=\alpha}\left(\mathcal{D}_{\alpha_{1}} \cdots \mathcal{D}_{\alpha_{j}}\right)$, for $\alpha \geq 2$. By also expressing $\Omega_{\alpha}(T)$ in frequency space using Eq. (3), the desired result follows by equating terms filtering the same term $O_{v_{1}} \cdots O_{v_{\alpha}} \otimes B_{u_{1}}\left(\omega_{1}\right) \cdots B_{u_{\alpha}}\left(\omega_{\alpha}\right)$.

Thanks to the above theorem, arbitrary generalized FFs are explicitly, non-recursively computable from the appropriate FFFs. In fact, FFFs suffice to fully characterize the cancellation and filtering capabilities that a control protocol can guarantee under minimal assumptions on the noise model. We will say that $G_{\vec{u} \vec{v}}^{(\alpha)}(\vec{\omega}, T)$ has generalized FO $\Phi_{\vec{u} \vec{v}}^{(\alpha)}$ and generalized CO $\Delta_{\vec{u} \vec{u}}^{(\alpha)}$ (around $\vec{\omega}=\vec{\omega}_{0}$ and $\left.T=0\right)$ if $G_{\vec{u} \vec{v}}^{(\alpha)}(\vec{\omega}, T) \sim$ $\mathcal{O}\left(m^{\Phi_{\bar{u} \hat{v}}^{(\alpha)}}\left(\vec{\omega}-\vec{\omega}_{0}\right) T^{\Delta_{\vec{u} \hat{v}}^{(\alpha)}+1}\right)$, where $m^{d}(\vec{x})$ denotes a degree$d$ monomial in the components of $\vec{x}$. One can similarly define fundamental FO and CO for each of the FFFs, say, $\phi_{\vec{u} \vec{v}}^{(\alpha)}$ and $\delta_{\vec{u} \vec{v}}^{(\alpha)}$, respectively. However, the contribution of operators resulting from $H_{e}(t)$ is filtered only by products of relevant generalized or fundamental FFs, for which $O_{v_{1}} \cdots O_{v_{\alpha}} \neq \mathbb{I}_{S}$ in at least one factor of such products, whereas all others correspond to pure-bath evolution $H_{B}^{\text {eff }}$ that is "irrelevant" for $S$. For each Magnus order $\alpha$, we denote the relevant set by $\mathcal{R}_{\alpha}$.

Definition. The generalized and fundamental $C O(\Delta, \delta)$ of a control protocol are given by the minimum of the corresponding quantity over the set of all the relevant FFs:

$$
\Delta=\min _{\mathcal{R}_{\alpha}, \forall \alpha}\left\{\Delta_{\vec{u} \vec{v}}^{(\alpha)}\right\}, \quad \delta=\min _{\mathcal{R}_{\alpha}, \forall \alpha}\left\{\delta_{\vec{u} \vec{v}}^{(\alpha)}\right\} .
$$

Similarly, the generalized and fundamental FO up to level $\kappa$, $\left(\Phi^{[\kappa]}, \phi^{[\kappa]}\right), \kappa=1, \ldots, \infty$, are obtained by minimizing over the set of all the relevant $F F$ s for $\alpha \leq \kappa$ :

$$
\Phi^{[\kappa]}=\min _{\mathcal{R}_{\alpha}, \alpha \leq \kappa}\left\{\Phi_{\vec{u} \vec{v}}^{(\alpha)}\right\}, \quad \phi^{[\kappa]}=\min _{\mathcal{R}_{\alpha}, \alpha \leq \kappa}\left\{\phi_{\vec{u} \vec{v}}^{(\alpha)}\right\} .
$$

When specific knowledge is available on the error model, we may further restrict the above minimizations to a smaller set of relevant FFs, yielding effective FOs or COs that may be higher than in Eqs. (7)-(8). For zero-mean Gaussian dephasing as previously discussed, for instance, since only even powers of $G^{(1)}(\omega, T)$ contribute to the reduced dynamics, it follows that 
$\Phi_{\text {eff }}^{[\infty]}=2 \Phi^{[\infty]}$, in line with the standard analysis based on $F_{p}(\omega, T) / \omega^{2}$. Thus, the quantities defined in Eqs. (7)-(8) yield lower bounds in general. The following result holds:

Theorem 2. The generalized and fundamental FO $\left(\Phi^{[\kappa]}, \phi^{[\kappa]}\right)$ and $C O(\Delta, \delta)$ obey the following relationships:

$$
\Phi^{[\kappa]}=\phi^{[\kappa]}, \quad \kappa=1, \ldots, \infty ; \quad \Delta=\delta ; \quad \phi^{[\infty]} \leq \delta .
$$

Proof. By expressing $G_{\vec{u} \vec{v}}^{(\alpha)}$ in terms of FFFs, Eq. $\sqrt{6}$, it follows that $\Phi_{\vec{u} \vec{v}}^{(\alpha)} \geq \min _{\mathcal{R}_{\alpha^{\prime} \leq \alpha}}\left\{\phi_{\vec{u} \vec{v}}^{\left(\alpha^{\prime}\right)}\right\}$, for each fixed $\alpha \geq 1$ and $(\vec{u}, \vec{v})$. Hence, minimizing the left hand-side yields $\Phi^{[\kappa]}=$ $\min _{\mathcal{R}_{\alpha \leq \kappa}} \Phi_{\vec{u} \vec{v}}^{(\alpha)} \geq \min _{\mathcal{R}_{\alpha^{\prime} \leq \kappa}}\left\{\phi_{\vec{u} \vec{v}}^{\left(\alpha^{\prime}\right)}\right\}=\phi^{[\kappa]}$. However, by inverting the relationship between Magnus and Dyson terms [17], one may also similarly express each $F_{\vec{u} \vec{v}}^{(\alpha)}$ in terms of generalized FFs. Thus, we also have $\Phi^{[\kappa]} \leq \phi^{[\kappa]}$, whereby the first equality in Eq. (9) follows. By a similar reasoning, minimizing over all $\mathcal{R}_{\alpha}$, the equality $\Delta=\delta$ also follows.

To establish the third inequality in Eq. (9), note that each $F_{\vec{u} \vec{v}}^{(\alpha)}$ has dimensions $\left[T^{\alpha}\right]$. By definition of the associated FO and CO, it must also be that $T^{\alpha} \sim T^{-\Phi_{\bar{u} \vec{v}}^{(\alpha)}+\Delta_{\bar{u} \vec{v}}^{(\alpha)}+1}$. Since by definition $\alpha \geq 1$, it thus follows that $\Phi_{\vec{u} \vec{v}}^{(\alpha)} \leq \Delta_{\vec{u} \vec{v}}^{(\alpha)}$. The final step is to minimize over $\mathcal{R}_{\alpha}$, for all $\alpha$.

Discussion.- In order to gain insight into the general results described above, we first consider single-axis control protocols - in particular, the ideal single-qubit DD setting as before, but now in the presence of arbitrary, non-Gaussian dephasing. In this case, it is well known that $\mathrm{CO} \delta$ over an evolution time $T$ may be achieved by using either (single-axis) concatenated DD [3] or Uhrig DD [4] - say, $\mathrm{CDD}_{\delta}$ and $\mathrm{UDD}_{\delta}$, respectively, with the latter protocol being optimal in terms of the required number of control pulses, also equal to $\delta$. For $\mathrm{CDD}_{\delta}$, thanks to the symmetry properties that the concatenated structure grants to both the FFFs of lower level and the control functions $y_{u v}(t)$, one may easily prove the following [22]:

Proposition 1. Arbitrarily high FO may be achieved for ideal single-axis DD via concatenation: $\phi^{[\infty]}=\delta$ for $\mathrm{CDD}_{\delta}$.

UDD protocols behave very differently. Recalling that no even-order FFF is relevant since $\sigma_{z}^{2}=\mathbb{I}_{S}$, we have explicitly computed the first odd FFFs for COs $\delta=1, \ldots, 8$. We find that $\phi_{z}^{(1)}=\delta, \phi_{z z z}^{(3)}=\delta-2$, and

$\phi_{z z z z z}^{(5)}=\left\{\begin{array}{l}\delta-2, \delta \in[3,4], \\ \delta-4, \delta \in[5,8],\end{array} \phi_{z z z z z z z}^{(7)}=\left\{\begin{array}{l}\delta-2, \delta \in[3,6], \\ \delta-6, \delta \in[7,8],\end{array}\right.\right.$

implying that the $\mathrm{FO} \phi^{[\infty]} \leq 1$ or 2 up to $\operatorname{CO} \delta=8$, depending of whether $\delta$ is odd or even. We conjecture that this holds for $\delta>8$. Thus, while both UDD and CDD ensure arbitrary CO, only the latter guarantees an arbitrary FO as well.

The implications of this difference may be appreciated by contrasting two dephasing toy models: in model $1, B$ consists of a qubit, such that $\tilde{H}_{e, 1}(t)=g y(t) \sigma_{z}^{(1)} \otimes\left[\cos (\omega t) \sigma_{z}^{(2)}+\right.$ $\left.\sin (\omega t) \sigma_{y}^{(2)}\right]$, whereas model 2 may be thought of as a classical version, with, say, $\tilde{H}_{e, 2}(t)=g y(t) \cos (\omega t) \sigma_{z}^{(1)}$, or

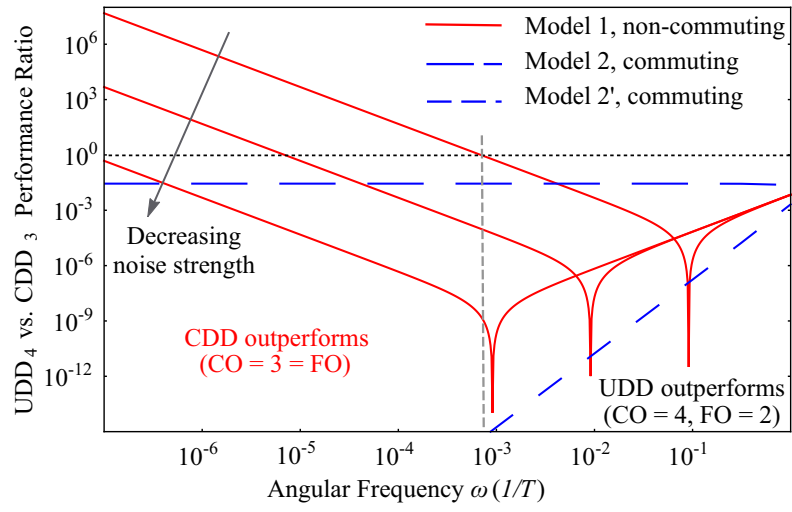

FIG. 1. (Color online) Performance of $\mathrm{CDD}_{3}$ and $\mathrm{UDD}_{4}$ for "commuting" (dashed lines) vs. "non-commuting" (solid lines) single-qubit dephasing models (see text). A performance ratio $\left\|H_{S B}^{\text {eff }}(T)\right\|_{\mathrm{UDD}_{4}} /\left\|H_{S B}^{\text {eff }}(T)\right\|_{\mathrm{CDD}_{3}}<1$ (black dotted line) implies that $\mathrm{UDD}_{4}$ outperforms $\mathrm{CDD}_{3}$. Only the latter model is sensitive to the difference in FO, which manifests in the change in slope of the solid lines in the low-frequency regime $\left(\omega \lesssim 10^{-3}\right)$. Parameters are $T=1$ and $g=9 / 40,9 / 400,9 / 4000$ in appropriate units, such that $\left\|\tilde{H}_{e, 1(2)}(t) T\right\|<1$ for all $t \in[0, T]$ and convergence is ensured.

$\tilde{H}_{e, 2}^{\prime}(t)=g y(t) \sin (\omega t) \sigma_{z}^{(1)}$. Here, $g$ is an overall coupling constant, and using a single frequency "tone" allows one to study the response to specific values of $\omega$. A comparison between $\mathrm{CDD}_{3}$ and $\mathrm{UDD}_{4}$ is given in Fig. 1, where in order to avoid making assumptions about the initial state of $S$ and $B$ and the statistical properties of the noise, we choose a performance metric inspired by fault-tolerance analysis [19], $\left\|T H_{S B}^{\text {eff }}(T)\right\|$, here computed via the Magnus expansion up to the third order. Due to its commuting nature, model 2 is (trivially) insensitive to the difference in FO. Thus, $\mathrm{UDD}_{4}$ always outperforms $\mathrm{CDD}_{3}$, since the higher $\mathrm{CO}$ is all that matters when a single FF is important. In contrast, since high-order FFs contribute to $\left\|T H_{S B}^{\text {eff }}(T)\right\|$ for model 1 , the difference in FO translates into the existence of a low-frequency regime in which $\mathrm{CDD}_{3}$ outperforms $\mathrm{UDD}_{4}$, despite its lower CO. Since the effect of the higher-order Magnus terms is reduced as $g$ decreases, this frequency range is, correspondingly, reduced. Despite its simplicity, this example thus clearly indicates that the FO can be the key property when the task is to remove noise that is stronger in a particular frequency range.

For noise models and/or control objectives requiring multiaxis protocols, aiming to arbitrarily high-order filtering is too stringent a requirement in general, even for a single qubit:

Proposition 2. A protocol which does not achieve perfect cancellation of an arbitrary quasi-static noise model (i.e., one where $\left\{B_{u}(t)\right\}$ are constant), has $\phi^{[\infty]}=0$.

Existing DD/DCG control sequences subject to a minimum switching-time constraint fall in this category (see [22] for a sketch of the proof). While we conjecture that the above "nogo" result may hold more generally for control protocols subject to physical constraints. the practical implication is that, in general, it is only meaningful to demand a high FO for a 
subset of the GFFs - e.g., those responsible for filtering the dominant noise contributions over a frequency range, that is, $\Phi^{[\kappa]}$ large for some $\kappa<\infty$. Remarkably, within the validity of a first-order fidelity approximation, the distinction between CO and such "truncated" $(\kappa=2)$ FO has been already observed for well-known composite-pulse protocols [5]: e.g., so-called Solavay-Kitaev SK1 and Wimperis BB1 sequences for amplitude errors have same FO $(=1)$ despite being firstand second-order in the Magnus sense, respectively [13, 15]. Likewise, Walsh-modulated logic gates with desired noisefiltering features against dephasing noise have been recently implemented in trapped-ion experiments [13].

Beyond the single-qubit setting, the FFF formalism presented here has proved instrumental to design DD protocols for multi-qubit dephasing that are more resource-efficient than existing ones [25]. While further investigation is needed to fully elucidate the significance of the noise-filtering perspective for dynamical quantum error control, we believe that our results provide a concrete starting point to meet this challenge.

We thank Paola Cappellaro, Alexandre Cooper-Roy, and Kaveh Khodjasteh for useful input, and Michael Biercuk for constructive feedback and a critical reading of the manuscript. Partially supported from the US ARO (contract No. W911NF11-1-0068) and the Constance and Walter Burke Special Projects Fund in Quantum Information Science.

[1] D. A. Lidar and T. A. Brun (eds.), Quantum Error Correction (Cambridge University Press, 2013).

[2] L. Viola and S. Lloyd, Phys. Rev. A 58, 2733 (1998); L. Viola, E. Knill, and S. Lloyd, Phys. Rev. Lett. 82, 2417 (1999).

[3] K. Khodjasteh and D. A. Lidar, Phys. Rev. Lett. 95, 180501 (2005).

[4] G. S. Uhrig, Phys. Rev. Lett. 98, 100504 (2007).

[5] J. T. Merrill and K. R. Brown, Adv. Chem. Phys. 154, 241 (2014).

[6] K. Khodjasteh and L. Viola, Phys. Rev. Lett. 102, 080501 (2009); K. Khodjasteh, D. A. Lidar, and L. Viola, ibid. 104, 090501 (2010); K. Khodjasteh, H. Bluhm, and L. Viola, Phys.
Rev. A 86, 042329 (2012).

[7] J. Bylander, S. Gustavsson, F. Yan, F. Yoshihara, K. Harrabi, G. Fitch, D. G. Cory, Y. Nakamura, J.-S. Tsai, and W. D. Oliver, Nature Phys. 7, 565 (2011); G. A. Álvarez and D. Suter, Phys. Rev. Lett. 107, 230501 (2011).

[8] A. Ajoy and P. Cappellaro, Phys. Rev. Lett. 110, 220503 (2013); A. Bootkatz, P. Wocjan, and L. Viola, New J. Phys. 16, 045021 (2014).

[9] A. G. Kofman and G. Kurizki, Phys. Rev. Lett. 87, 270405 (2001).

[10] L. Cywiński, R. M. Lutchyn, C. P. Nave, and S. Das Sarma, Phys. Rev. B 77, 174509 (2008).

[11] D. Hayes, K. Khodjasteh, L. Viola, and M. J. Biercuk, Phys. Rev. A 84, 062323 (2011).

[12] M. J. Biercuk, A. C. Doherty, and H. Uys, J. Phys. B 44, 154002 (2011); T. J. Green, H. Uys, and M. J. Biercuk, Phys. Rev. Lett. 109, 020501 (2012); T. J. Green, J. Sastrawan, H. Uys, and M. J. Biercuk, New J. Phys. 15, 095004 (2013).

[13] A. Soare, H. Ball, M. C. Jarratt, J. J. McLoughlin, X. Zhen, T. J. Green, and M. J. Biercuk, arXiv:1404.0820 (2014).

[14] L. Bouten, R. Van Handel, and M. R. James, SIAM J. Control Optim. 46, 2199, (2007); J. Gough and M. R. James, Commun. Math. Phys. 287, 1109 (2009).

[15] C. Kabytayev, T. J. Green, K. Khodjasteh, M. J. Biercuk, L. Viola, and K. R. Brown, Phys. Rev. A 90, 012316 (2014).

[16] W. M. Witzel, K. Young, and S. Das Sarma, arXiv:1307.2597.

[17] S. Blanes, F. Casas, J. A. Oteo, and J. Ros, Phys. Rep. 470, 151 (2009).

[18] S. Gustavsson, F. Yan, J. Bylander, F. Yoshihara, Y. Nakamura, T. P. Orlando, and W. D. Oliver, Phys. Rev. Lett. 109, 010502 (2012).

[19] J. Preskill, Quantum Inf. Comput. 13, 181 (2013).

[20] H. K. Ng, D. A. Lidar, and J. Preskill, Phys. Rev. A 84, 012305 (2011).

[21] C. L. Nikias and J. M. Mendel, IEEE Signal Proc. Magazine 93, 10 (1993).

[22] See supplementary material for additional technical detail.

[23] R. Kubo, J. Phys. Soc. Japan 17, 1100 (1962).

[24] Mathematically, it is essential for the GFF picture to be valid that high-order noise cumulants (equivalently, correlation functions) in the time domain obey appropriate analyticity assumptions, ensuring that power spectra decay sufficiently fast at high frequencies. See D. Stanek, B. Fauseweh, C. Stihl, S. Pasini, and G. S. Uhrig, arXiv:1404.3836, for a counter-example.

[25] G. A. Paz-Silva, S.-W. Lee, T. J. Green, and L. Viola, forthcoming.

\section{SUPPLEMENTARY INFORMATION}

\section{The simplest example: Single-qubit single-axis control}

Consider a single-qubit model system, in which the internal Hamiltonian $H_{S}$ may be taken to be zero (in a suitable frame) and noise acts only along a known axis, chosen here to be the $z$ axis. Assume that control is implemented in terms of sequences of perfect instantaneous (bang-bang) $\pi$ pulses, and that the control objective is to achieve $\operatorname{DD}\left(Q=\mathbb{I}_{S}\right)$. In the interaction picture with respect to the bath Hamiltonian, $H_{B}$, we may write

$$
\tilde{H}_{e}(t)=y(t) \sigma_{z} \otimes B(t),
$$

where for simplicity we have denoted the control switching function $y_{z z}(t) \equiv y(t)$ and, likewise, $B_{z}(t) \equiv B(t)$ [cf. Eq. (1) in the main text]. The error propagator induced by such Hamiltonian after an evolution time $T$ is given by

$$
\tilde{U}_{e}(T)=\mathcal{T}_{+} e^{-i \int_{0}^{T} \tilde{H}_{e}(t) d t}=e^{-i T\left(H_{S B}^{\mathrm{eff}}(T)+H_{B}^{\mathrm{eff}}(T)\right)},
$$


where $\mathcal{T}_{+}$denotes the time-ordering operator. By taking advantage of the fact that $\left(\sigma_{z}\right)^{2 m}=\mathbb{I}_{S}$, we may express the relevant error-action contributions in terms of the generalized FFs and the Fourier transforms of $B(t)$ defined in the main text [Eq. (3)]:

$$
\begin{aligned}
T H_{S B}^{\mathrm{eff}}(T) & =\sigma_{z} \otimes \sum_{\alpha: \text { odd }} G^{(\alpha)}(\vec{\omega}, T) B\left(\omega_{1}\right) \cdots B\left(\omega_{\alpha}\right), \\
T H_{B}^{\mathrm{eff}}(T) & =\sum_{\alpha: \text { even }} G^{(\alpha)}(\vec{\omega}, T) B\left(\omega_{1}\right) \cdots B\left(\omega_{\alpha}\right) .
\end{aligned}
$$

It is worth highlighting that since we are working in the interaction picture with respect to $H_{B}$, the error Hamiltonian $H_{e}(t)$ is explicitly time-dependent even before moving to the control toggling-frame, and hence the traditional definition of decoupling order, $\delta$, as determined by the lowest-order Magnus term which acts solely on the bath, does not apply. In fact, this is evident from a direct calculation in the presence of spin-boson dephasing in the interaction picture, see for example [4]: even the first Magnus term has a non-trivial action on the system, and yet decoherence suppression is still achieved. Notice however, that the main consequence of demanding the first $\delta$ Magnus terms to be pure-bath in the standard toggling-frame setting, and what is truly essential for achieving decoherence suppression, is that the norm of the residual system-bath interaction is sufficiently small: that is, we require $\left\|T H_{S B}^{\text {eff }}(T)\right\| \sim \mathcal{O}\left(T^{\delta+1}\right)$ for order- $\delta$ DD, thus yielding an interaction-picture evolution $U(T)=\tilde{U}_{e}(T)=$ $\mathbb{I}_{S} \otimes U_{B}(T)+\mathcal{O}\left(T^{\delta+1}\right)$, for some unitary pure-bath operator $U_{B}(T)$. Defining, as in the main text, the cancellation order $(\mathrm{CO})$ in this way is natural when working in the interaction picture or, more generally, whenever one has a time-dependent classical or quantum noise source, thereby formally allowing for a unified treatment. We also refer to $\mathrm{Ng}$ et al. [20] for additional discussion of $\mathrm{DD}$ in the interaction picture.

Back to the problem at hand, we wish to determine the qubit reduced density matrix at time $T, \rho_{S}(T)$, specified by matrix elements $\rho_{\ell m}(T)$ with respect to the $z$ basis. By assuming an initial factorized state, $\rho_{S B}(0)=\rho_{S}(0) \otimes \rho_{B}$ (this assumption can in fact be weakened, as we shall further discuss in a future work), and using Eq. (3) above, we have:

$$
\begin{aligned}
\rho_{\ell m}(T) & =\left\langle\ell\left|\operatorname{Tr}_{B}\left[\tilde{U}_{e}(\tau) \rho_{S}(0) \otimes \rho_{B} \tilde{U}_{e}^{\dagger}(T)\right]\right| m\right\rangle \\
& =\rho_{\ell m}(0) \operatorname{Tr}_{B}\left[e^{-i\left[(-1)^{\ell}-(-1)^{m}\right] \sum_{\alpha: \text { odd }} \int \frac{d^{\alpha} \vec{\omega}^{\alpha}}{(2 \pi)^{\alpha}} G^{(\alpha)}(\vec{\omega}, T) B\left(\omega_{1}\right) \cdots B\left(\omega_{\alpha}\right)} \rho_{B}\right] .
\end{aligned}
$$

The partial trace in square brackets can be evaluated using the following generalized cumulant expansion [23]. By letting $\langle\cdot\rangle_{q} \equiv \operatorname{Tr}_{B}\left[\cdot \rho_{B}\right]$, the cumulant expansion of $\left\langle e^{P}\right\rangle_{q}$, where $P$ is a generic bounded linear operator, is given by

$$
\left\langle e^{P}\right\rangle_{q}=e^{\sum_{k=1}^{\infty} \frac{C^{(k)}\left(P^{k}\right)}{k !}}
$$

where $C^{(k)}\left(P^{k}\right)$ is the $k$-th cumulant of $P$, which is assumed to be linear in its arguments (i.e., $C^{(k)}\left(P_{1}^{k}+P_{2}^{k}\right)=C^{(k)}\left(P_{1}^{k}\right)+$ $C^{(k)}\left(P_{2}^{k}\right)$ ). By performing a symbolic expansion of the exponentials on both sides of the equality and associating $C^{(k)}\left(P^{k}\right)$ with terms where $P$ appears $k$ times, one finds that cumulants can be expressed in terms of high-order moments $\langle\cdot\rangle_{q}$, much in the same way as in classical statistical mechanics they are written in terms of standard high-order moments, $\langle\cdot\rangle_{c}[23]$. Of course, the treatment is equally valid if one replaces the quantum bath with a classical bath. In that case, as noted in the main text, we may let $B(t)=\beta(t) \mathbb{I}_{B}$, with $\beta(t)$ being a stochastic process and $\langle\cdot\rangle_{q}$ being replaced by the corresponding classical expectation, $\langle\cdot\rangle_{c}$, Because of the infinite sum in the exponent of the right hand side of Eq $[6$, writing a closed-form solution or getting useful insight is not always straightforward, but some cases of interest allow considerable simplifications. In particular, we consider two such scenarios here: (i) Gaussian (quantum or classical) dephasing noise; and (ii) arbitrary classical dephasing noise.

Gaussian dephasing.- A Gaussian process strongly constrains the high-order cumulants, allowing us to truncate the infinite sum and get an exact solution. Classically, a noise process $\beta(t)$ is said to be Gaussian if $C^{(k)}\left(b\left(t_{1}\right) \cdots b\left(t_{k}\right)\right)=0$ for $k>2$, and of zero-mean if $C^{(k=1)}(b(t))=0$ [23]. In order to generalize this feature to the quantum case, one needs to be able to define the cumulant $C^{(k)}\left(B\left(t_{1}\right) \cdots B\left(t_{k}\right)\right)$ in such a way that the non-commuting character of $B(t)$ at different times is properly taken into account. This can be done in a straightforward fashion by considering $P=B\left(t_{1}\right)+\ldots+B\left(t_{k}\right)$ and using the above generalized cumulant expansion, Eq. (6). By identifying terms where the operators $\left\{B\left(t_{i}\right)\right\}$ appear in the fixed order within each cumulant, one can easily write any $C^{(k)}\left(B\left(t_{1}\right) \cdots B\left(t_{k}\right)\right)$ in terms of $\langle\cdot\rangle_{q}$, and vice versa. For example, after the formal expansion of the exponentials in Eq. (6), the "order $P^{3}$ " equation yields

$$
\left\langle P^{3}\right\rangle_{q}=C^{(3)}\left(P^{3}\right)+3 C^{(2)}\left(P^{2}\right) C^{(1)}(P)+C^{(1)}(P) C^{(1)}(P) C^{(1)}(P),
$$


and the linearity of the cumulants allows the identifications

$$
\begin{aligned}
\left\langle B\left(t_{2}\right) B\left(t_{3}\right) B\left(t_{1}\right)\right\rangle_{q}= & C^{(3)}\left(B\left(t_{2}\right) B\left(t_{3}\right) B\left(t_{1}\right)\right)+C^{(1)}\left(B\left(t_{2}\right)\right) C^{(1)}\left(B\left(t_{3}\right)\right) C^{(1)}\left(B\left(t_{1}\right)\right) \\
& +C^{(2)}\left(B\left(t_{2}\right) B\left(t_{3}\right)\right) C^{(1)}\left(B\left(t_{1}\right)\right)+C^{(2)}\left(B\left(t_{2}\right) B\left(t_{1}\right)\right) C^{(1)}\left(B\left(t_{3}\right)\right) \\
& +C^{(2)}\left(B\left(t_{3}\right) B\left(t_{1}\right)\right) C^{(1)}\left(B\left(t_{2}\right)\right), \\
\left\langle B\left(t_{3}\right) B\left(t_{1}\right) B\left(t_{2}\right)\right\rangle_{q}= & C^{(3)}\left(B\left(t_{3}\right) B\left(t_{1}\right) B\left(t_{2}\right)\right)+C^{(1)}\left(B\left(t_{3}\right)\right) C^{(1)}\left(B\left(t_{1}\right)\right) C^{(1)}\left(B\left(t_{2}\right)\right) \\
& +C^{(2)}\left(B\left(t_{3}\right) B\left(t_{1}\right)\right) C^{(1)}\left(B\left(t_{2}\right)\right)+C^{(2)}\left(B\left(t_{3}\right) B\left(t_{2}\right)\right) C^{(1)}\left(B\left(t_{1}\right)\right) \\
& +C^{(2)}\left(B\left(t_{1}\right) B\left(t_{2}\right)\right) C^{(1)}\left(B\left(t_{3}\right)\right) .
\end{aligned}
$$

As expected, when arbitrary operators $B(t)$ s at different times commute, both expressions are the same and one recovers the same relations one has for a classical noise process [23]. Since in the quantum case $\langle\cdot\rangle_{q}$ is a function of the initial state of the bath $\rho_{B}$, we say that the pair $\left\{B(t), \rho_{B}\right\}$ defines a quantum Gaussian noise process if $C^{(k)}\left(B\left(t_{1}\right) \cdots B\left(t_{k}\right)\right)=0$ for $k>2$ and, furthermore, the process has zero-mean when $C^{(k=1)}(B(t))=0$. The well-known linear spin-boson dephasing model falls in this category whenever the initial state of the bath is thermal, see e.g. [19, 25].

Thus, when the noise is Gaussian (classical or quantum) and has zero-mean, the expression for the reduced dynamics, Eq. (5), drastically simplifies and we get

$$
\begin{aligned}
\rho_{01}(T) & =\rho_{01}(0) e^{\frac{1}{2}\left\langle\left(-2 i \int_{-\infty}^{\infty} \frac{d \omega}{2 \pi} G^{(1)}(\omega, T) B(\omega)\right)^{2}\right\rangle} \\
& =\rho_{01}(0) e^{-2 \int_{-\infty}^{\infty} \frac{d \omega_{1}}{2 \pi} \int_{-\infty}^{\infty} \frac{d \omega_{2}}{2 \pi} G^{(1)}\left(\omega_{1}, T\right) G^{(1)}\left(\omega_{2}, T\right)\left\langle B\left(\omega_{1}\right) B\left(\omega_{2}\right)\right\rangle},
\end{aligned}
$$

with $\langle\cdot\rangle$ now denoting either $\langle\cdot\rangle_{c}$ or $\langle\cdot\rangle_{q}$. This yields the expression quoted in the main text for the decay rate $\chi_{p}(T)$ under the additional assumption that the noise is stationary: in such a case, by definition it must be $\left\langle B\left(t_{1}\right) B\left(t_{2}\right)\right\rangle=\left\langle B(t) B\left(t+t_{2}-t_{1}\right)\right\rangle$ for all $t$, whereby it follows that

$$
\left\langle B\left(\omega_{1}\right) B\left(\omega_{2}\right)\right\rangle \equiv S^{(2)}\left(\omega_{1}, \omega_{2}\right)=2 \pi \delta\left(\omega_{1}+\omega_{2}\right) S\left(\omega_{2}\right) .
$$

As noted in the main text, the relevant $\mathrm{FF} G^{(1)}(\omega, T)$ does not contribute directly in this case, but rather only through bilinear products $G^{(1)}\left(\omega_{1}, T\right) G^{(1)}\left(\omega_{2}, T\right)$. More general (e.g. non-zero mean) dephasing models may be similarly handled.

Arbitrary classical dephasing.- When the noise is classical, i.e., $B(t)=\beta(t) \mathbb{I}_{B}$, the structure of the equations can also be simplified. In this case, because all the higher-order commutators in the Magnus expansion vanish, the relevant ensemble average is given by

$$
\begin{aligned}
\left\langle\rho_{\ell m}(T)\right\rangle_{c} & =\left\langle\left\langle\ell\left|\tilde{U}_{e}(T) \rho_{S}(0) \tilde{U}_{e}^{\dagger}(T)\right| m\right\rangle\right\rangle_{c} \\
& =\rho_{\ell m}(0)\left\langle e^{-i\left[(-1)^{\ell}-(-1)^{m}\right] \int \frac{d \omega}{2 \pi} G^{(1)}(\omega, T) \beta(\omega)}\right\rangle_{c} \\
& =\rho_{\ell m}(0) e^{\sum_{k=1}^{\infty} \frac{\left\{i\left[(-1)^{m}-(-1)^{\ell}\right]\right\}^{k}}{k !} \int \frac{d^{k} \omega^{\omega}}{(2 \pi)^{k}} G^{(1)}\left(\omega_{1}, T\right) \cdots G^{(1)}\left(\omega_{k}, T\right)\left\langle\beta\left(\omega_{1}\right) \cdots \beta\left(\omega_{k}\right)\right\rangle_{c}} .
\end{aligned}
$$

Thus, similarly to the Gaussian case, all the effects of the control are condensed in a single FF, $G^{(1)}(\omega, T) \equiv F^{(1)}(\omega, \tau)$. However, products of arbitrary order may now be relevant in principle, depending on the non-Gaussian character of the noise statistics. It is worth highlighting that a similar simplification occurs in a dephasing (quantum) spin-boson noise model given a generic (non-Gaussian) initial bath state. In this case, higher order Magnus terms are also irrelevant, because they either vanish based on algebraic considerations or do not otherwise contribute to the reduced system dynamics [25].

\section{Fundamental FFs as building blocks: Single-qubit multi-axis control}

In the main text we showed that FFFs condense the filtering effect of a dynamical quantum error suppression protocol at the effective Hamiltonian and reduced dynamics level. It follows that this is also true for any functional of the propagator or the reduced density matrix of the system. To illustrate this claim and make contact with previous relevant work, we explicitly show how the "effective" generalized FFs introduced by Green et al. [12] can be rewritten in terms of the generalized FFs $\left\{G_{\vec{u} \vec{v}}^{(\alpha)}(\vec{\omega}, T)\right\}$ we introduced [Eqs. (3) and (6) in the main text].

In Refs. [12], the authors consider a (drift-less, $H_{S}=0$ ) single qubit exposed to a multi-axis classical noise process such that, using the notation of the main text, the time-dependent error Hamiltonian in the toggling frame with respect to the control may be written as

$$
\tilde{H}_{e}(t)=\sum_{u, v} y_{u v}(t) \sigma_{v} \beta_{u}(t), \quad u, v \in\{x, y, z\}
$$


where the stochastic process $\beta_{u}(t)$ describes classical noise in the $u$ spatial direction and $y_{u v}(t) \equiv[Y(t)]_{u v}=$ $\left(Y_{x}(t), Y_{y}(t), Y_{z}(t)\right)^{T} \in S O(3)$ is the relevant control matrix. If the propagator $U(T)$ resulting from the evolution induced by $H_{\text {ctrl }}(t)$ over a time $T$ would ideally execute a target gate $Q$, then, writing the total propagator in the presence of $H_{e}(t)$ as $U(T)=Q \tilde{U}_{e}(T)$ as in the main text, one can quantify the accuracy of a control operation subject to the noise process $\left\{\beta_{u}(t)\right\}$ via the operational (ensemble-averaged) fidelity

$$
\mathcal{F}_{\text {av }}(T)=\frac{1}{4}\left\langle\left|\operatorname{Tr}\left(\tilde{U}_{e}(T)\right)\right|^{2}\right\rangle
$$

One may write the error propagator $\tilde{U}_{e}(T)=\exp [-i \vec{a}(T) \cdot \vec{\sigma}]$, where $\vec{\sigma}=\left\{\sigma_{x}, \sigma_{y}, \sigma_{z}\right\}$ and $\vec{a}(T) \equiv a(T) \hat{a}(T)=\sum_{\alpha=1}^{\infty}[\vec{a}]_{\alpha}(T)$ is the "error vector" (in the terminology of [12]), computable via the Magnus expansion. Specifically, each $[\vec{a}]_{\alpha}(T)$ originates from the $\alpha$-th order Magnus term and the component associated to the operator $\sigma_{v}$ is determined by

$$
\left[a_{v}\right]_{\alpha}(T) \sigma_{v}=\sum_{\substack{\vec{u}, \vec{v} \text { such that } \\ \sigma_{v_{1}} \cdots \sigma_{v_{\alpha}}=\sigma_{v}}} \int_{-\infty}^{\infty} \frac{d \omega_{1}}{2 \pi} \cdots \int_{-\infty}^{\infty} \frac{d \omega_{\alpha}}{2 \pi} G_{\vec{u}, \vec{v}}^{(\alpha)}(\vec{\omega}, T) \sigma_{v_{1}} \cdots \sigma_{v_{\alpha}} \beta_{u_{1}}\left(\omega_{1}\right) \cdots \beta_{u_{\alpha}}\left(\omega_{\alpha}\right) .
$$

By re-expressing the fidelity in terms of the length $a(T)$ of the error vector, $\mathcal{F}_{\text {av }}(T)=\frac{1}{2}[\langle\cos (2 a)\rangle+1]$, Eq. $\sqrt{12}$ may be used as the starting point to obtain a fidelity expansion in terms of the generalized FFs and noise spectral density functions. For instance, in the relevant case of dephasing noise, whereby only $\beta_{z}(t)$ is non vanishing, the elements of the control matrix are $y_{z x}(t), y_{z y}(t)$ and $y_{z z}(t)$. By considering a Taylor expansion of the cosine term, the lowest order contributions to the fidelity are given by [12](b):

$$
\mathcal{F}_{\mathrm{av}}(T) \simeq 1-\sum_{v=x, y, z}\left\langle\left[a_{v}\right]_{1}^{2}\right\rangle-\left\{\sum_{v=x, y, z}\left(\left\langle\left[a_{v}\right]_{2}^{2}\right\rangle+2\left\langle\left[a_{v}\right]_{3}\left[a_{v}\right]_{1}\right\rangle\right)-\frac{1}{3} \sum_{v, v^{\prime}}\left\langle\left[a_{v}\right]_{1}^{2}\left[a_{v^{\prime}}\right]_{1}^{2}\right\rangle\right\} .
$$

This expression can be written in terms of our generalized FFs $\left\{G_{\vec{u} \vec{v}}^{(\alpha)}(\vec{\omega}, T)\right\}$, by using Eq. 12 , e.g.:

$$
\begin{aligned}
& {\left[a_{v}\right]_{1}(T)=\int_{-\infty}^{\infty} \frac{d \omega}{2 \pi} G_{v}^{(1)}(\omega, T) \beta_{z}(\omega), \quad v=x, y, z,} \\
& {\left[a_{x}\right]_{2}(T)=\int_{-\infty}^{\infty} \frac{d \omega_{1}}{2 \pi} \int_{-\infty}^{\infty} \frac{d \omega_{2}}{2 \pi} i\left(G_{y, z}^{(2)}\left(\omega_{1}, \omega_{2}, T\right)-G_{z, y}^{(2)}\left(\omega_{1}, \omega_{2}, T\right)\right) \beta_{z}\left(\omega_{1}\right) \beta_{z}\left(\omega_{2}\right),} \\
& {\left[a_{y}\right]_{2}(T)=\int_{-\infty}^{\infty} \frac{d \omega_{1}}{2 \pi} \int_{-\infty}^{\infty} \frac{d \omega_{2}}{2 \pi} i\left(G_{z, x}^{(2)}\left(\omega_{1}, \omega_{2}, T\right)-G_{x, z}^{(2)}\left(\omega_{1}, \omega_{2}, T\right)\right) \beta_{z}\left(\omega_{1}\right) \beta_{z}\left(\omega_{2}\right),} \\
& {\left[a_{z}\right]_{2}(T)=\int_{-\infty}^{\infty} \frac{d \omega_{1}}{2 \pi} \int_{-\infty}^{\infty} \frac{d \omega_{2}}{2 \pi} i\left(G_{x, y}^{(2)}\left(\omega_{1}, \omega_{2}, T\right)-G_{y, x}^{(2)}\left(\omega_{1}, \omega_{2}, T\right)\right) \beta_{z}\left(\omega_{1}\right) \beta_{z}\left(\omega_{2}\right),}
\end{aligned}
$$

and so on. Under the additional assumptions that the noise is stationary Gaussian and has zero mean, the above expressions can be further simplified. Since

$$
\begin{aligned}
\left\langle\beta_{z}\left(\omega_{1}\right) \beta_{z}\left(\omega_{2}\right) \beta_{z}\left(\omega_{3}\right) \beta_{z}\left(\omega_{4}\right)\right\rangle= & \left\langle\beta_{z}\left(\omega_{1}\right) \beta_{z}\left(\omega_{2}\right)\right\rangle\left\langle\beta_{z}\left(\omega_{3}\right) \beta_{z}\left(\omega_{4}\right)\right\rangle+\left\langle\beta_{z}\left(\omega_{1}\right) \beta_{z}\left(\omega_{3}\right)\right\rangle\left\langle\beta_{z}\left(\omega_{2}\right) \beta_{z}\left(\omega_{4}\right)\right\rangle \\
& +\left\langle\beta_{z}\left(\omega_{1}\right) \beta_{z}\left(\omega_{4}\right)\right\rangle\left\langle\beta_{z}\left(\omega_{2}\right) \beta_{z}\left(\omega_{3}\right)\right\rangle
\end{aligned}
$$

the statistical properties of the noise are entirely captured by the power spectral density, $S_{z}(\omega)=\int_{-\infty}^{\infty} d t e^{-i \omega \tau}\left\langle\beta_{z}(t) \beta_{z}(t+\tau)\right\rangle$. One can then rewrite Eq. [13] in the frequency domain (see in particular Eq. (4) in the Supplementary Material of [12](b)):

$$
\mathcal{F}_{\mathrm{av}}(T) \simeq 1-\frac{1}{4 \pi} \int_{0}^{\infty} \frac{d \omega}{\omega^{2}} S_{z}(\omega) F_{1}(\omega, T)-\frac{1}{(4 \pi)^{2}} \sum_{p} \int_{0}^{\infty} d \omega S_{z}(\omega) \int_{0}^{\infty} \frac{d \omega^{\prime}}{{\omega^{\prime}}^{2}} S_{z}\left(\omega^{\prime}\right) F_{p, 2}\left(\omega, \omega^{\prime}, T\right),
$$

where $F_{1}(\omega, T)$ and $F_{p, 2}\left(\omega, \omega^{\prime}, \tau\right)$ are, in our context, effective generalized FFs computed through $\left\{\left[a_{v}\right]_{\alpha}\right\}$, and the index $p$ generically refers to higher-order contributions. While we shall not proceed to further rewrite all their equations in terms of our $\left\{G_{\vec{u} \vec{v}}^{(\alpha)}(\vec{\omega}, T)\right\}$ here, it should be clear at this point that $\left\{G_{\vec{u} \vec{v}}^{(\alpha)}(\vec{\omega}, \tau)\right\}$, and by extension $\left\{F_{\vec{u} \vec{v}}^{(\alpha)}(\vec{\omega}, T)\right\}$, appear as building blocks of the final expressions for the effective generalized FFs found in Ref. [12]. For instance, it is easy to verify that

$$
\frac{F_{1}(\omega, T)}{\omega^{2}}=F_{x}^{(1)}(\omega, T) F_{x}^{(1)}(-\omega, T)+F_{z}^{(1)}(\omega, \tau) F_{z}^{(1)}(-\omega, T)+F_{y}^{(1)}(\omega, T) F_{y}^{(1)}(-\omega, T)
$$

generalizing the correspondence $F_{p}(\omega, T) / \omega^{2}=F^{(1)}(\omega, T) F^{(1)}(-\omega, T)$ already discussed for single-axis control under dephasing noise. 


\section{Proof of Proposition 1}

We wish to show that, by assuming access to perfect instantaneous control pulses (say, along the $x$ axis), a FO $\phi^{[\infty]}=\delta$ is achieved by single-axis DD after $\delta$ levels of concatenation, for arbitrary dephasing noise on a qubit - as claimed in the main text.

The controlled evolution under a $\mathrm{CDD}_{k}$ protocol, with $\mathrm{CO}$ equal to $k$, can be obtained via the recursion

$$
U_{k+1}\left(T_{k+1}=2 T_{k}\right)=\sigma_{x} U_{k}\left(T_{k}\right) \sigma_{x} U_{k}\left(T_{k}\right),
$$

where $U_{0}\left(T_{0}\right)$ denotes free evolution under a general dephasing Hamiltonian of the form $H=\sigma_{z} \otimes B+H_{B}$ over a time $T_{0}$. In the toggling frame associated to the control pulses and the interaction picture with respect to $H_{B}$, the joint dynamics is ruled by the Hamiltonian already given in Eq. 11 in the Supplement, $\tilde{H}_{e}(t)=y(t) \sigma_{z} \otimes B(t)$, and $\mathrm{CO}=\delta$ means that the resulting interaction-picture propagator obeys

$$
U_{\delta}\left(T_{\delta}\right)=\mathbb{I}_{S} \otimes U_{B}\left(T_{\delta}\right)+\mathcal{O}\left(T_{\delta}^{\delta+1}\right) .
$$

We will show that this also achieves $\phi^{[\infty]}=\delta$. Recalling Eqs. (3)-(4) in the Supplement, the FFFs of interests are

$$
F^{(\alpha)}\left(\vec{\omega}, T_{\delta}\right) \equiv F_{k=\delta}^{(\alpha)},
$$

and in particular the ones with odd Magnus index $\alpha$, since those are associated with terms in $H_{S B}^{\text {eff }}\left(T_{\delta}\right)$.

The starting point is to show that $F_{k=1}^{(\alpha)}$ has FO $\phi_{k=1}^{(\alpha)} \geq 1$ for all $\alpha$. Since $U_{1}\left(T_{1}\right)=\sigma_{x} U_{0}\left(T_{0}\right) \sigma_{x} U_{0}\left(T_{0}\right)$, note that $y(t)=+1$ for $t \in\left[0, T_{0}\right]$ and $y(t)=-1$ for $t \in\left[T_{0}, 2 T_{0}\right]$, that is, the control switching function obeys the symmetry property $y(t)=-y\left(t+T_{0}\right)$. Exploiting this symmetry and dividing the length- $T_{1}$ integration intervals in subintervals of length $T_{0}$, one finds that the $\mathcal{O}\left(\omega^{0}\right)$ contribution to $F_{k=1}^{(\alpha)}$, obtained by letting $\vec{\omega}=0$ in the expression for $F^{(\alpha)}\left(\vec{\omega}, T_{1}\right)$, is given by

$$
\begin{aligned}
\left.F_{k=1}^{(\alpha)}\right|_{0} & =\int_{0}^{T_{1}} d s_{1} \int_{0}^{s_{1}} d s_{2} \cdots \int_{0}^{s_{\alpha}} d s_{\alpha} y\left(s_{1}\right) \cdots y\left(s_{\alpha}\right) \\
& =\left.\left[1+(-1)^{\alpha}\right] F_{k=0}^{(\alpha)}\right|_{0}+\left.\left.\sum_{a=1}^{\alpha-1}(-1)^{a} F_{k=0}^{(a)}\right|_{0} F_{k=0}^{(\alpha-a)}\right|_{0} \\
& =\left[1+(-1)^{\alpha}\right] \frac{T_{0}^{\alpha}}{\alpha !}+\sum_{a=1}^{\alpha-1}(-1)^{a} \frac{T_{0}^{a}}{a !} \frac{T_{0}^{\alpha-a}}{(\alpha-a) !} \\
& =\frac{\left(T_{0}-T_{0}\right)^{\alpha}}{\alpha !}=0 .
\end{aligned}
$$

Hence, $\phi_{k=1}^{(\alpha)} \geq 1$ for all $\alpha$. To proceed, we first observe that, as a consequence of the recursion in Eq. [16, it also follows that $y(t)=-y\left(t+T_{\delta-1}\right)$ for $t \in\left[0, T_{\delta-1}\right]$. Again, we exploit this symmetry by decomposing each FFF appropriately. Using the definition

$$
I_{q, q+1}^{l_{1}, l_{2}}\left(T_{\delta-1}\right) \equiv \int_{q T_{\delta-1}}^{(q+1) T_{\delta-1}} d s_{l_{1}} \int_{q T_{\delta-1}}^{s_{l_{1}}} d s_{l_{1}+1} \ldots \int_{q T_{\delta-1}}^{s_{l_{2}-1}} d s_{l_{2}} e^{i \sum_{r} \omega_{r} s_{r}} y\left(s_{l_{1}}\right) \ldots y\left(s_{l_{2}}\right)
$$

one finds that

$$
\begin{aligned}
F_{k=\delta}^{(\alpha)} & =\int_{0}^{T_{\delta}} d s_{1} \int_{0}^{s_{1}} d s_{2} \cdots \int_{0}^{s_{\alpha-1}} d s_{\alpha} e^{i \sum_{r} \omega_{r} s_{r}} y\left(s_{1}\right) \ldots y\left(s_{j}\right), \\
& =I_{0,1}^{1, \alpha}\left(T_{\delta-1}\right)+I_{1,2}^{1, \alpha}\left(T_{\delta-1}\right)+\sum_{1 \leq a<\alpha} I_{1,2}^{1, a}\left(T_{\delta-1}\right) \cdot I_{0,1}^{a+1, \alpha}\left(T_{\delta-1}\right) \\
& =\left[1+(-1)^{\alpha} e^{i \sum_{r} \omega_{r} T_{\delta-1}}\right] I_{0,1}^{1, \alpha}\left(T_{\delta-1}\right)+\sum_{1 \leq a<\alpha} I_{1,2}^{1, a}\left(T_{\delta-1}\right) \cdot I_{0,1}^{a+1, \alpha}\left(T_{\delta-1}\right) .
\end{aligned}
$$

Now, if $\alpha$ is odd, either $a$ or $\alpha-a$ are odd (but not both) and $\left[1+(-1)^{\alpha} e^{i \sum_{r} \omega_{r} T_{\delta-1}}\right] \sim \mathcal{O}\left(m^{1}(\vec{\omega}) T_{\delta-1}\right)$, whereas if $\alpha$ is even, then $a$ and $\alpha-a$ must both be either odd or even and $\left[1+(-1)^{\alpha} e^{i \sum_{r} \omega_{r} T_{\delta-1}}\right] \sim \mathcal{O}\left(m^{0}(\vec{\omega})\right)$. Notice that $I_{q, q+1}^{1, a}\left(T_{\delta-1}\right)$ and $I_{q, q+1}^{a+1, \alpha}\left(T_{\delta-1}\right)$ are proportional to $F^{(a)}\left(\vec{\omega}, T_{\delta-1}\right)$ and $F^{(\alpha-a)}\left(\vec{\omega}, T_{\delta-1}\right)$, respectively. Then, since the FO of the left hand side of Eq. 20 is lower-bounded by the FO of each term on the right hand side, the following recursion holds:

$$
\begin{aligned}
\phi_{k=\delta+1}^{(\alpha: \text { even })} \geq \min \left[\phi_{k=\delta}^{(\alpha: \text { :even })},\left\{\phi_{k=\delta}^{(a)}+\phi_{k=\delta}^{(\alpha-a)}\right\}_{a<\alpha}\right] \\
\phi_{k=\delta+1}^{(\alpha: \text { odd })} \geq \min \left[\phi_{k=\delta}^{(\alpha: \text { odd })}+1,\left\{\phi_{k=\delta}^{(a)}+\phi_{k=\delta}^{(\alpha-a)}\right\}_{\text {a:odd }<\alpha}\right] .
\end{aligned}
$$

Since we have already shown that $\phi_{k=1}^{(\alpha)} \geq 1$ for all $\alpha$, inductive reasoning implies that $\phi_{k=\delta}^{(\alpha: \text { even })}=1$ and $\phi_{k=\delta}^{(\alpha: \text { odd })}=\delta$, from which the desired result follows. 


\section{Proof of Proposition 2}

We wish to show that if a protocol does not achieve perfect cancellation of an arbitrary quasi-static noise model, then it must have infinite-level FO $\phi^{[\infty]}=0$. We prove it by contradiction.

Assume that the protocol in consideration, defined by the control matrix $\left\{y_{u v}(t)\right\}$, does not achieve perfect cancellation of a quasi-static noise model but has $\phi^{[\infty]}>0$. Then one would have that $F_{\vec{u} \vec{v}}^{(\alpha)}(\vec{\omega}, T)$ is at least $\mathcal{O}\left(\omega^{1}\right)$, and thus it must be that (letting $\omega_{j}=0 \forall j$ in Eq. (5) of the main text)

$$
\int_{0}^{T} d t_{1} \int_{0}^{t_{1}} d t_{2} \cdots \int_{0}^{t_{\alpha-1}} d t_{\alpha} y_{u_{1} v_{1}}\left(t_{1}\right) \cdots y_{u_{\alpha} v_{\alpha}}\left(t_{\alpha}\right)=0
$$

for all relevant $\alpha$ and $\vec{u}, \vec{v}$, that is, such that $O_{v_{1}} \cdots O_{v_{\alpha}} \neq \mathbb{I}_{S}$. However, the integral in the left hand-side of Eq. (23) is precisely the integral appearing in every Magnus term when applying the control sequence to a quasi-static noise model, namely, one where every relevant bath operator $B_{u}(t)$ is constant. Thus, if a protocol has $\phi^{[\infty]}>0$ for an arbitrary (potentially unknown) noise model, then it must also be capable of perfect cancellation of any (again, potentially unknown) quasi-static noise model. If so, the resulting (interaction-picture) propagator is exactly of the form $U(T)=\mathbb{I}_{S} \otimes U_{B}(T)$. Since by hypothesis cancellation is not, however, achieved perfectly, we have reached a contradiction. This implies that for any control protocol which does not achieve perfect cancellation of an arbitrary quasi-static error model, there must exist (at least) a relevant $F_{\vec{u}, \vec{v}}^{(\alpha)}$ such that $\phi_{\vec{u} \vec{v}}^{(\alpha)}=0$, thus forcing $\phi^{[\infty]}=0$.

As mentioned in the main text, we conjecture that a stronger no-go results does in fact hold, namely, that the infinite-level FO $\phi^{[\infty]}=0$ for arbitrary open-loop control protocols subject to a physical minimum-switching time constraint. Additional discussion and rigorous derivations will be presented elsewhere. 